IDDF2019-ABS-0244 PERCUTANEOUS NEEDLE ASPIRATION VS. PIGTAIL CATHETER DRAINAGE IN AMOEBIC LIVER ABSCESS: REPORT FROM A SINGLE CENTRE IN MINING AREA OF INDIA

Jayant Ghosh*, Sujata Kujur, Pragya Pant, Rash Kujur. Orchid Medical Centre, Ranchi, Jharkhand, India

\subsection{6/gutjnl-2019-IDDFabstracts.293}

Background Amoebic Liver Abscess (ALA) is one of the most common infectious diseases of the liver in mining areas of India due to the widespread consumption of alcohol. Although mortality rate has come down from $90 \%$ in the early 20th century to $10 \%$, still ALA leads to significant morbidity, absence from work, and expenditures. Treatment of large ALA could be done either by Percutaneous Needle Aspiration (PNA) or Percutaneous Catheter Drainage (PCD) or Surgery.

Methods It was a retrospective study done over three years period from October 2015 up to September 2018 in all patients with ALA $(>5 \mathrm{~cm})$ who underwent either PNA or PCD.

Results Total of 37 patients' data were found; however, only 32 could be analysed as incomplete data was present in the rest 5 patients. Eighteen patients underwent PNA and 14 underwent PCD. Clinical recovery was found in both (PNA vs. PCD) groups in almost comparable time ( 21 days vs. 18 days; $\mathrm{p}=0.1$ ). However, we had given antibiotics in both groups for 28 days as per our protocol. One patient developed percutaneous sinus tract was formed as a major complication in PCD group, however, no complications were found in PNA group. In PNA group one patient underwent surgery due to non-resolving abscess, ongoing severe sepsis, and multi-organ failure. No mortality was observed in any groups. However, the total cost of therapy was significantly low in PNA group as compared to PCD group (266.67\$ vs. $400 \$)$.

\begin{tabular}{llll}
$\begin{array}{l}\text { Abstract IDDF2019-ABS-0244 Table } \\
\text { PNA and PCD groups }\end{array}$ & Comparison between \\
\hline Parameters & PNA & PCD & p-value \\
\hline Clinical recovery (days) & 21 & 18 & 0.1 \\
Average duration of antibiotic therapy (i.v. plus oral) & 28 & 28 & As per \\
(days) & & & protocol \\
Complications & 0 & 1 & 0.7 \\
Required Surgery & 1 & 1 & 0.5 \\
Mortality & 0 & 0 & 0.5 \\
Cost of Therapy (\$) & 266.67 & 400 & $<0.01$ \\
\hline
\end{tabular}

Conclusions PNA with antibiotics should be the first option in poor resource areas in the management of large ALA.

\section{IDDF2019-ABS-0247 GLOBAL TEMPORAL PATTERN OF PANCREATIC CANCER: AN UPDATED ANALYSIS}

Junjie Huang ${ }^{*}$, Veeleah Lok, Colette Leung, Paul Chan, Jingxuan Wang, Martin Wong. Jockey Club School of Public Health and Primary Care, Faculty of Medicine, The Chinese University of Hong Kong, Hong Kong

\subsection{6/gutjnl-2019-IDDFabstracts.294}

Background Pancreatic cancer induces a substantial global burden. We have previously evaluated its temporal trend in the period 1998-2007. With new data available, this study aimed to update its global incidence/mortality trends in the past decade.
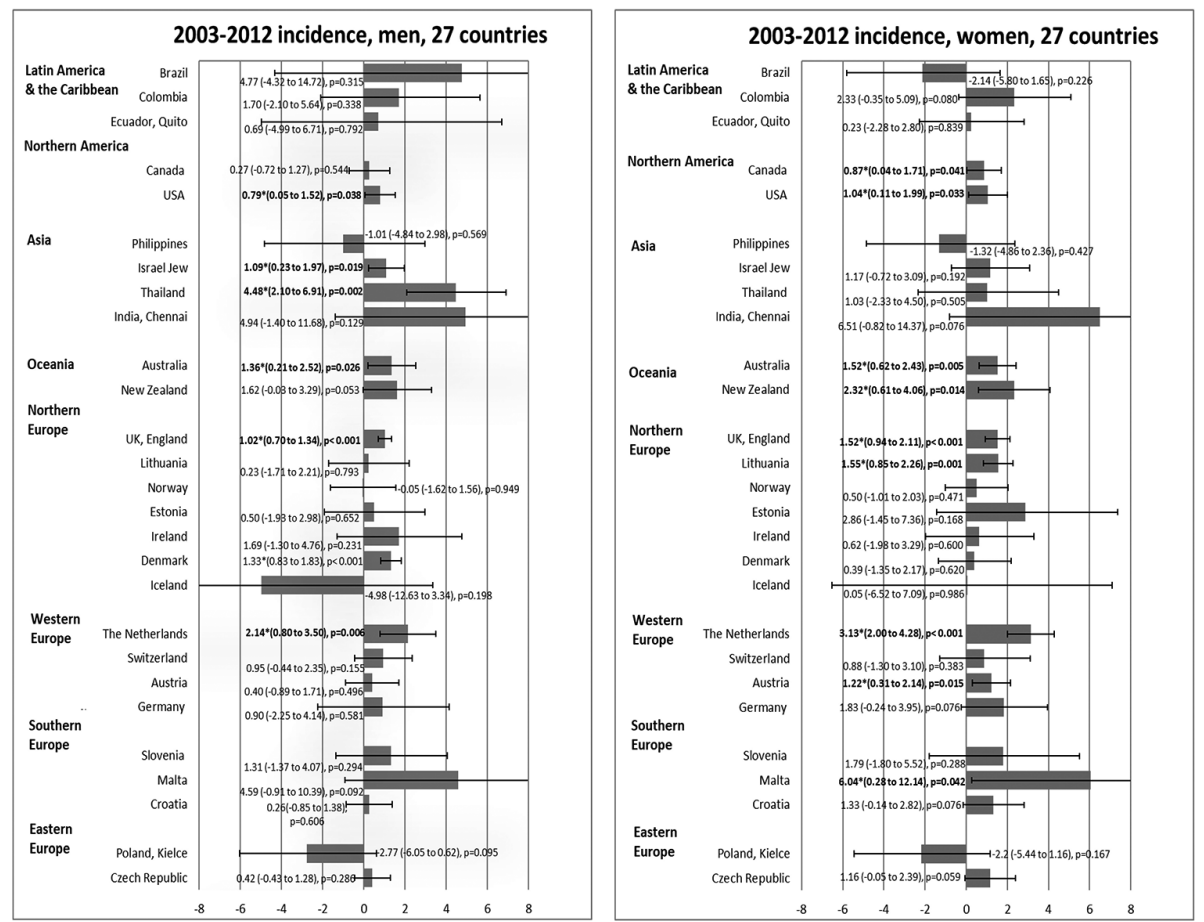

Abstract IDDF2019-ABS-0247 Figure 1 The average annual percentage change AAPC of pancreatic cancer incidence in men left and women right 2003-2012 